\title{
O CUSTO MÉDIO DIRETO DO MATERIAL UTILIZADO EM CIRURGIA DE REVASCULARIZAÇÃO DO MIOCÁRDIO
}

Eliana Bittar*, Valéria Castilho

Trabalho realizado no Instituto Dante Pazzanese de Cardiologia, São Paulo, SP.

RESUMO - Objetivos. Verificar o custo médio direto do material usado em cirurgia de revascularização do miocárdio e comparar 0 custo médio encontrado segundo o número de pontes de safena realizadas.

Métodos. Como referencial teórico para apuração dos custos utilizou-se 0 sistema de custeio de absorção por produto/procedimento. A pesquisa, do tipo descritiva, foi realizada no centro cirúrgico de um hospital especializado em cardiologia, no município de São Paulo. A amostra foi conformada por 104 cirurgias de revascularização do miocárdio.

Resultados. 0 levantamento do consumo possibilitou aferição do custo médio direto de material usado em cirurgia de revascularização do miocárdio que resultou em $\mathbf{R} \$ 2.718,78$.
ConclusöEs. Houve uma variação de custos do material, em relação ao número de pontes de safena realizadas, havendo uma diferença significativa entre as cirurgias de I, 2 e 3 pontes, o que não ocorreu entre as cirurgias de 3,4 e 5 pontes. 0 custo médio direto das cirurgias foi: I ponte (R\$2.207,7I), 2 pontes (R\$2.554,6I), 3 pontes (R\$2.768.94), 4 pontes $(\mathbf{R} \$ 2.848,65)$ e 5 pontes $(\mathbf{R} \$ 2.884,13)$. Os itens de material de perfusão ( $R \$ 1.051,24)$, fios cirúrgicos $(\mathbf{R} \$ 829,98)$ e material de consumo $(\mathbf{R} \$ 442,40)$ foram os que apresentaram 0 maior custo médio.

Unitermos: Enfermagem cardiológica. Cirurgia cardíaca. Revascularização miocárdica. Custos. Economia Médica. Economia da Saúde.

\section{INTRODUÇÃo}

Em um cenário globalizado, competitivo e de rápidas mudanças, os administradores e os funcionários, no dia-a-dia, são desafiados a rever suas ações e propor novas formas e meios de gerenciar suas organizações, para aumentar a lucratividade e garantir a sobrevivência.

As inúmeras dificuldades de ordem econômico-financeiras decorrentes de uma conjuntura nacional extremamente complexa vêm tornar o custo um tema essencial no gerenciamento das organizações tanto privadas como públicas, com fins lucrativos ou mesmo filantrópicos'.

Dentro da área de saúde, o compromisso em relação ao estudo dos custos e ao aumento de lucratividade das organizaçōes não deveria ser diferente, no entanto, observa-se que os profissionais da área de saúde tendem a rejeitar qualquer aproximação com esses problemas administrativos, o que tem colocado os

\footnotetext{
* Correspondência:

Av. Dr. Dante Pazzanese 500

Ibirapuera - Caixa postal 215 - CEP:04012-909

Tel: 50854153 e 50854116 bittar@usp.br
}

hospitais em condições delicadas, com dificuldades de sobrevivência. Essa rejeição tem como premissa a idéia de que controlar o custo da assistência nas organizações de saúde pode acarretar em diminuição de recursos e, conseqüentemente, perda de qualidade.

Conhecer os custos dos serviços de saúdeé importante, pois permite identificar os pontos da organização que precisam ser reduzidos e isto se faz controlando gastos, eliminando desperdícios, trabalhando com eficiência e preservando a qualidade no atendimento prestado ${ }^{2,3}$.

A equipe de enfermagem desempenha um importante papel relacionado ao material de consumo, por ser usuária de uma quantidade considerável dos mesmos ${ }^{4}$. Assim, torna-se notória a necessidade de conhecimento por parte da equipe sobre o custo do material que utiliza, evitando o aumento do custo pelo mau uso do equipamento ou por desperdício. Desperdício é a falta de qualidade no serviço prestado pelo uso inadequado do recurso usado, fazendo seu custo elevar. Dessa maneira, julga-se importante o enfermeiro transmitir à sua equipe uma consciência da importância do custo na assistência, a fim de conseguir o uso adequado de material e equipamento, bem como evitar desperdícios ${ }^{5}$.
Trabalhando há mais de dez anos em um hospital público, como enfermeira de centro cirúrgico, tenho observado o desconhecimento e a falta de envolvimento dos profissionais de saúde nas questões relativas ao controle do consumo de material nos procedimentos cirúrgicos e quanto isso representa monetariamente à instituição.

Nos hospitais públicos, os procedimentos cirúrgicos são pagos pelo Ministério da Saúde (MS), mediante uma tabela de preços com valores fechados. Em razão do fato de não terem um sistema de custeio efetivo, não se pode saber se o valor recebido pelo MS cobre o custo real de cada procedimento.

Existe um estudo em que foi analisado o custo direto no ato operatório de revascularização do miocárdio e utilizou-se para o controle de consumo dos insumos, "Kits" padronizados 6 . Mas, em minha prática diária, não dispomos no centro cirúrgico de um apoio para seu preparo, acondicionamento e, ainda, para efetuar o estorno do material não utilizado nas cirurgias; portanto para realização do nosso estudo, utilizamos a Planilha de Consumo de Material em Cirurgia de Revascularização do Miocárdio. 
Diante dessa realidade, o nosso objetivo é calcular o custo direto do material utilizado em cirurgia de revascularização do miocárdio e comparar o custo médio direto do material, segundo o número de pontes de safena realizadas.

\section{Métodos}

Trata-se de uma pesquisa descritiva, assim, tendo sido analisado o consumo e o custo direto do material utilizado em cirurgia de revascularização do miocárdio, por meio do registro dos dados na Planilha de Consumo de Material em Cirurgia de Revascularização do Miocárdio. Não foram inclú́dos outros itens de material como: instrumentais cirúrgicos e roupas esterilizadas, que dependeriam de cálculos de rateio de outras unidades da instituição.

Para o desenvolvimento do estudo, optou-se pelo sistema de custeio de absorção por produto/procedimento, como método de apuração de custos ${ }^{7}$.

\section{Local de estudo}

A pesquisa foi desenvolvida no centro cirúrgico do Instituto Dante Pazzanese de Cardiologia (IDPC), especializado em cirurgia cardiovascular, que atende pacientes do Sistema Único de Saúde (SUS) e convênios. Trata-se de um hospital governamental subordinado à Coordenadoria de Saúde da região metropolitana da Grande São Paulo.

\section{População e amostra}

A população do estudo corresponde às cirurgias eletivas de revascularização do miocárdio com circulação extra-corpórea. Este procedimento cirúrgico é o mais freqüente no centro cirúrgico da Instituição do estudo, tendo em média 60 procedimentos mensais e 720 anuais.

A amostra foi constituída por todos os procedimentos realizados no período de janeiro a fevereiro de 200I, totalizando 104 procedimentos, sendo excluídas as cirurgias que ultrapassaram o horário das 19 horas, pela dificuldade da pesquisadora estar acompanhando os auxiliares de enfermagem na coleta de dados, no período noturno.

\section{Instrumento de coleta de dados}

Para a coleta de dados, foi utilizada a Planilha de Consumo de Material em Cirurgia de Revascularização do Miocárdio.

Tabela I - Custo direto do material utilizado em cirurgia de revascularização do miocárdio (moeda real e moeda dólar). São Paulo, 200I

$\begin{array}{llllll}\text { Moedareal } & & & & & \\ \text { Custodiretodo } & \text { Média } & \text { Mediana } & \text { DP } & \text { Mínimo } & \text { Máximo } \\ \text { Materialemreal } & 2.718,78 & 2.758,84 & 536,80 & 1.723,43 & 4.373,76 \\ \text { DP = Desvio padrão } & & & & & \end{array}$

Intervalo de confiança $(95,0 \%)=[2.615,60 ; 2.822,00]$

Moedadólar*

Custodiretodo Média $\quad$ Mediana DP $\quad$ Mínimo Máximo

$\begin{array}{llllll}\text { materialem dólar } & 1.329,35 & 1.348,93 & 262,46 & 842,67 & 2.138,54\end{array}$

$\mathrm{DP}=$ Desvio padrão

* Dólar comercial = R\$2,0452-Fevereiro 200I

Intervalo de confiança $(95,0 \%)=[1.278,89 ; 1.379,8 I]$

\section{Procedimento de coleta de dados}

Após a aprovação da Comissão Científica do Instituto Dante Pazzanese de Cardiologia os dados da Planilha de Consumo de Material em Cirurgia de Revascularização do Miocárdio foram coletados pelos dez auxiliares de enfermagem dos turnos matutino e vespertino, sob a supervisão e orientação da pesquisadora.

\section{Tratamento e análise dos dados}

As informações coletadas foram organizadas em um banco de dados, em formato Excel. A análise dos dados foi realizada por intermédio do programa SPSS por Windows, versão 8.0

Com o intuito de investigar a influência do número de pontes de safena realizadas sobre o custo da cirurgia, estas foram classificadas em três grupos: grupo I (I ou 2 pontes), grupo || (3 pontes) e grupo III (4 ou 5 pontes). Para comparar o custo médio entre os três grupos, foi usada a técnica de Análise de Variância (ANOVA) ${ }^{8}$. O método empregado para localizar as diferenças, duas a duas, foi o de comparações múltiplas de Tukey ${ }^{8}$.

Os resultados cujos níveis descritivos (valores de p) foram inferiores a 0,05 , foram considerados estatisticamente significativos.

A moeda corrente usada para o cálculo dos custos foi a unidade monetária brasileira denominada real e a moeda americana dólar. Nos meses de janeiro e fevereiro, período da coleta de dados, a cotação do dólar comercial variou de $R \$ 1,9384$ a $R \$ 2,0452$.

\section{Resultados}

A Tabela I refere-se ao custo direto do material de revascularização do miocárdio.

Os dados da Tabela I demonstram o custo médio direto do material utilizado em cirurgia de revascularização do miocárdio, igual a $R \$ 2.718,78$ ou US\$1.329,35, o custo mínimo igual a $R \$ 1.723,43$ ou US\$842,67 e o custo máximo igual a $\mathrm{R} \$ 4.373,76$ ou US\$2.138,54. Para efeito do cálculo do custo do material, utilizou-se o intervalo de confiança de $95,0 \%$ que resultou em um valor entre $R \$ 2.615,60$ ou U\$1.278,89 à $R \$ 2.822,00$ ou U\$1.379,8I.

$\mathrm{Na}$ Tabela 2, visualizamos o custo direto da cirurgia de revascularização do miocárdio, segundo tipo de material.

A Tabela 2 mostra o custo por item de material utilizado em cirurgia de revascularização do miocárdio e faz perceber que 0 material de perfusão $(P)$ foi o item cujo custo médio apresentou-se mais elevado, seguido do item fios cirúrgicos $(\mathrm{FC})$ e, em seguida, vem o item material de consumo (MC). Oitem cujo custo médio foi o mais baixo foi derivados de sangue (DS).

A Tabela 3 nos mostra a freqüência da cirurgia, por número de pontes de safena realizadas.

Como demonstraram os dados da Tabela 3 houve predominância das cirurgias de 3 pontes (31,7\%), seguida de 4 pontes $(27,8 \%), 2$ pontes $(16,3 \%), 5$ pontes $(14,4 \%)$ e I ponte $(9,6 \%)$. 
A Tabela 4 nos mostra o custo direto do material utilizado em cirurgia de revascularização do miocárdio, por número de pontes de safena.

Os dados da Tabela 4 demonstraram que houve uma variação do custo de material utilizado e que 0 custo da cirurgia aumenta de acordo com o aumento do número de pontes de safena realizadas. Pode-se observar na Tabela 4 o cálculo do coeficiente de variação (C.V.), que compara o desvio padrão com a média. $O$ coeficiente de variação menor ou igual a 0,25 indica que o conjunto de dados é razoavelmente homogêneo ${ }^{9}$. Com isso, pode-se observar na Tabela 4 um coeficiente de variação inferior a 0,25 demonstrando ser uma amostra homogênea.

A Tabela 5 refere-se ao custo da cirurgia de revascularização domiocárdio, segundo tipo do material por número de pontes de safena.

Observa-se nos dados da Tabela 5 que, independente do número de pontes de safena realizadas, o item de material que apresenta o custo mais elevado é o de perfusão $(P)$, seguido de fios cirúrgicos (FC) e material de consumo (MC), e o item que apresentou o custo mais baixo foi derivados de sangue (DS).

$\mathrm{Na}$ Tabela 6, visualizamos o custo direto do material utilizado na cirurgia de revascularização do miocárdio, nos grupos I, II e III (I ou 2, 3 e 4 ou 5 pontes de safena).

A Tabela 6 mostra que o custo da cirurgia aumenta de acordo com o aumento do número de pontes de safena, quando analisado em grupo. Da mesma maneira, quando éanalisado por número de pontes de safena individualmente na Tabela 4, podemos observar também um coeficiente de variação inferior $\mathrm{a} 0,25$, nos três grupos, significando ser uma amostra homogênea?.

A Tabela 7 compara o custo médio entre os três grupos (I ou 2, 3 e 4 ou 5 pontes de safena), para isso foi utilizado a técnica da analise de variância - ANOVA.

AANOVA indicou diferenças significativas entre os três grupos, ou seja, o custo da cirurgia depende do número de pontes realizadas. Nas comparações duas a duas, verifica-se que não houve diferenças significativas entre o custo médio de cirurgias de 3 pontes e de cirurgias de 4 ou 5 pontes (diferença média de $\mathrm{R} \$ 91,80)$. 0 custo

\begin{tabular}{lccccc}
\hline \multicolumn{5}{c}{ Tabela 2 - Custo direto da cirurgia de revascularização do miocárdio, } \\
segundo tipo de material. São Paulo, 200I \\
\hline Material & Média & Mediana & DP & Mínimo & Máximo \\
Perfusão & $1.051,24$ & $1.134,57$ & 308,60 & 705,25 & $1.477,37$ \\
Fioscirúrgicos & 829,98 & 785,69 & 262,42 & 422,18 & $2.059,39$ \\
Materialdeconsumo & 442,40 & 433,51 & 48,95 & 350,98 & 649,18 \\
Medicação & 149,65 & 103,80 & 112,79 & 51,67 & 737,09 \\
Equipamentos & 89,79 & 85,23 & 15,29 & 63,67 & 120,37 \\
Soros & 59,84 & 57,58 & 14,55 & 32,45 & 108,20 \\
Soluções & 44,16 & 40,83 & 17,16 & 17,46 & 114,17 \\
Gases & 40,75 & 38,69 & 7,22 & 28,92 & 61,90 \\
Derivadosdesangue & 10,97 & 0,00 & 13,86 & 0,00 & 54,96 \\
\hline
\end{tabular}

$\mathrm{DP}=$ Desvio padrão

Tabela 3 - Número total de cirurgias de revascularização do miocárdio, distribuída por número de pontes de safena. São Paulo, 200I

\begin{tabular}{ccc}
\hline № de pontes de safena * & Total de cirurgias & $\%$ \\
1 & 10 & 9,6 \\
2 & 17 & 16,3 \\
3 & 33 & 31,7 \\
4 & 29 & 27,8 \\
5 & 15 & 14,4 \\
Total & 104 & 100,0 \\
\hline
\end{tabular}

*Quandose menciona número de pontes de safena, inclui-se todos os tipos de enxertos arteriais(mamáriae radial)e enxertosvenosos(safena).

Tabela 4 - Custo direto do material utilizado em cirurgia de revascularização do miocárdio, por número de pontes de safena (moeda real e moeda dólar). São Paulo, 2001

\begin{tabular}{cccccccc}
\hline $\begin{array}{c}\text { Moeda real } \\
\text { No }\end{array}$ & $\begin{array}{c}\text { Média } \\
\text { pontes }\end{array}$ & $\begin{array}{c}\text { Mediana } \\
\text { R }\end{array}$ & $\begin{array}{c}\text { DP } \\
\text { R }\end{array}$ & $\begin{array}{c}\text { Mínimo } \\
\text { R\$ }\end{array}$ & $\begin{array}{c}\text { Máximo } \\
\text { R\$ }\end{array}$ & $\mathbf{n}$ & CV \\
2 & $2.207,71$ & $2.128,15$ & 323,69 & $1.842,35$ & $2.883,18$ & 10 & 0,15 \\
3 & $2.554,61$ & $2.429,99$ & 515,79 & $1.723,43$ & $3.452,43$ & 17 & 0,20 \\
4 & $2.768,94$ & 2.907 .20 & 463,70 & $1.964,80$ & $3.670,97$ & 33 & 0,17 \\
5 & $2.848,65$ & $3.026,23$ & 576,13 & $2.017,86$ & $4.373,76$ & 29 & 0,20 \\
& $2.884,13$ & $2.798,94$ & 551,18 & $2.058,98$ & $4.097,77$ & 15 & 0,19 \\
\hline
\end{tabular}

$\mathrm{DP}=$ Desvio padrão; $\mathrm{CV}=$ Coeficiente de variação; $n$ = número de cirurgias

\begin{tabular}{cccccccc}
$\begin{array}{c}\text { Moeda dólar* } \\
\text { No }\end{array}$ & Média & Mediana & DP & Mínimo & Máximo & n & CV \\
pontes & US\$ & US\$ & US\$ & US\$ & US\$ & & \\
1 & $1.079,45$ & $1.040,55$ & 158,26 & 900,81 & $1.419,73$ & 10 & 0,15 \\
2 & $1.249,07$ & $1.188,14$ & 252,19 & 842,67 & $1.688,06$ & 17 & 0,20 \\
3 & $1.353,87$ & $1.421,47$ & 226,70 & 960,68 & $1.794,91$ & 33 & 0,17 \\
4 & $1.392,84$ & $1.479,67$ & 281,69 & 986,63 & $2.138,54$ & 29 & 0,20 \\
5 & $1.410,19$ & $1.368,54$ & 269,49 & $1.006,73$ & $2.003,60$ & 15 & 0,19 \\
\hline
\end{tabular}

$\mathrm{DP}=$ Desvio padrão

$\mathrm{CV}=$ Coeficientede variação

$\mathrm{n}=$ número de cirurgias

* Dólar comercial $=$ R $\$ 2,0452-$ Fevereiro 2001 
Tabela 5 - Custo direto da cirurgia de revascularização do miocárdio, segundo o tipo de material, por número de pontes de safena. São Paulo, 200I

\begin{tabular}{|c|c|c|c|c|c|c|c|c|c|c|}
\hline \multirow[t]{3}{*}{ Material } & \multicolumn{8}{|c|}{ Número de pontes de safena } & \multirow{2}{*}{\multicolumn{2}{|c|}{5}} \\
\hline & \multicolumn{2}{|c|}{ I } & \multicolumn{2}{|c|}{2} & \multicolumn{2}{|c|}{3} & \multicolumn{2}{|c|}{4} & & \\
\hline & Média & DP & Média & DP & Média & DP & Média & DP & Média & DP \\
\hline Perfusão & 888,5 & 223,0 & 978,7 & 406,8 & $1.112,0$ & 286,9 & $1.097,0$ & 290,0 & $1.021,0$ & 289,7 \\
\hline Fios cirúrgicos & 599,9 & 151,0 & 744,6 & 164,9 & 813,1 & 199,6 & 907,4 & 315,4 & 967,3 & 301,5 \\
\hline Material de consumo & $4 \mid 3,3$ & 38,8 & 462,1 & 54,5 & 447,9 & 55,6 & 436,4 & 40,5 & 438,7 & 40,7 \\
\hline Medicação & 98,5 & 58,3 & $127, \mid$ & 84,3 & 159,4 & $|26|$, & 144,3 & 82,6 & 198,1 & $|67|$, \\
\hline Equipamentos & 63,8 & 0,5 & 80,8 & 2,2 & 83,9 & 8,5 & 98,7 & 8,4 & 112,9 & 2,2 \\
\hline Soros & 53,7 & 10,0 & 64,5 & 18,2 & 57,9 & 13,7 & 63,0 & 15,9 & 56,5 & 8,8 \\
\hline Soluçōes & 50,0 & $|4|$, & 39,6 & 8,6 & 42,3 & 15,2 & 51,7 & 2,7 & 34,6 & 10,5 \\
\hline Gases & 35,5 & 5,7 & 39,3 & 7,6 & 40,2 & 7,1 & 41,7 & 7,2 & 44,1 & 6,2 \\
\hline Derivados sangue & $4, I$ & 6,6 & 16,9 & 16,4 & 12,0 & 12,7 & 8,5 & $\mid 4,4$ & 10,9 & 13,9 \\
\hline
\end{tabular}

$\mathrm{DP}=$ Desvio padrão

Tabela 6 - Custo direto do material utilizado em cirurgia de revascularizacão do miocárdio, nos grupos de I ou 2 pontes, 3 pontes e 4 ou 5 pontes de safena. São Paulo, 200 I

\begin{tabular}{cccccccc}
\hline № pontes & Média & Mediana & DP & Mínimo & Máximo & $\mathbf{n}$ & CV \\
I ou 2 & $2.426,13$ & $2.292,24$ & 478,67 & $1.723,43$ & $3.452,43$ & 27 & 0,19 \\
3 & $2.768,94$ & $2.907,20$ & 463,70 & $1.964,80$ & $3.670,97$ & 33 & 0,16 \\
4 ou 5 & $2.860,74$ & $2.832,48$ & 561,55 & $2.017,86$ & $4.373,76$ & 44 & 0,19
\end{tabular}

$\mathrm{DP}=$ Desvio padrão

$\mathrm{n}=$ número de cirurgias

\begin{tabular}{lccc}
\hline \multicolumn{4}{c}{ Tabela 7 - Técnica de análise de variância - ANOVA. São Paulo, 200I } \\
\hline Comparação & Teste de Tukey & Diferença média & I.C. $(95,0 \%)$ \\
& (p-valor) & (em R\$) & p/ diferença \\
custo $_{1-2} \times$ custo $_{3}$ & 0,030 & 342,80 & {$[27,2 ; 658,4]$} \\
custo $_{1-2}$ xusto $_{4-5}$ & 0,002 & 434,60 & {$[137,3 ; 731,9]$} \\
custo $_{3} \times$ custo $_{4-5}$ & 0,716 & 91,80 & {$[-188,2 ; 371,9]$} \\
\hline
\end{tabular}

Tabela 8 - Custo direto da cirurgia de revascularização do miocárdio, segundo tipo de material nos grupos de I ou 2 pontes, 3 pontes e 4 ou 5 pontes de safena. São Paulo, 200I

\begin{tabular}{|c|c|c|c|c|c|c|c|}
\hline \multirow{3}{*}{$\begin{array}{l}\text { Material } \\
\text { Grupos }\end{array}$} & \multicolumn{6}{|c|}{ Números de pontes de safena } & \multirow{3}{*}{$\begin{array}{l}\text { ANOVA } \\
\text { (p-valor) }\end{array}$} \\
\hline & \multicolumn{2}{|c|}{ I. I ou 2} & \multicolumn{2}{|c|}{ II -3} & \multicolumn{2}{|c|}{ III - 4 ou 5} & \\
\hline & Média & DP & Média & DP & Média & DP & \\
\hline Perfusão & 945,3 & 347,8 & I.III,7 & 286,9 & $1.070,7$ & 288,8 & 0,098 \\
\hline Fios cirúrgicos & 691,0 & 422,1 & 813,1 & 519,8 & 927,8 & 308,5 & 0,001 \\
\hline Material de consumo & 444,0 & 54,1 & 447,9 & 55,6 & 437,1 & 40,0 & 0,622 \\
\hline Medicação & 116,5 & 75,8 & 159,4 & $|26|$, & 162,6 & 119,2 & 0,207 \\
\hline Equipamentos & 74,5 & 8,5 & 83,9 & 8,5 & 103,5 & 9,7 & 0,001 \\
\hline Soros & 60,5 & 16,3 & 57,9 & 13,7 & 60,8 & $|4|$, & 0,671 \\
\hline Soluções & 43,5 & 11,9 & 42,3 & 15,2 & 45,9 & 21,0 & 0,649 \\
\hline Gases & 38,3 & 7,2 & 40,2 & 7,1 & 42,6 & 6,9 & 0,046 \\
\hline Derivados de sangue & 12,2 & 14,9 & 12,0 & 12,7 & 9,3 & $|4|$, & 0,606 \\
\hline
\end{tabular}

$\mathrm{DP}=$ Desvio padrão médio das cirurgias de I ou 2 pontes foi significativamente menor que o custo das cirurgias de 3 e 4 ou 5 pontes. Em média, as cirurgias de 3 pontes custam $R \$ 342,80$ a mais que as cirurgias de I ou 2 pontes. Quanto às cirurgias de 4 ou 5 pontes, esta diferença média é $R \$ 434,60$.

A Tabela 8 refere-se ao custo de cirurgia de revascularização do miocárdio, segundo tipo de material nos grupos I, II e III (I ou 2,3 e 4 ou 5 pontes de safena).

Pode-se afirmar quando o item material é analisado em grupo, independente do número de pontes de safena realizadas, o que apresenta o custo médio mais elevado é o material de perfusão $(P)$, seguido de fios cirúrgicos $(F C)$ e material de consumo (MC). Afirma-se, também, que os itens fios cirúrgicos $(\mathrm{FC})$, gases $(\mathrm{G})$ e equipamentos ( $E Q$ ) aumentam seu custo em ordem crescente de grupol, Il e III, ou seja, de acordo com o aumento do número de pontes de safena realizadas, e apresentaram valores de $p<0,05$, portanto estatisticamente significativos. Quando o item medicações é analisado em grupos, há um aumento de seu custo em relação ao acréscimo do número de ponte de safena realizada, diferente quando é analisado por número de pontes de safena isoladas. Os demais itens da planilha independem do aumento dos números de pontes realizadas nos grupos I, II e III.

\section{Discussão}

Verificou-se que o custo médio do material utilizado em cirurgia de revascularização do miocárdio foi $R \$ 2.718,78$, no entanto, 
pôde-se perceber que só o item perfusão, apresentou um custo médio de $R \$ 1.051,24 \mathrm{e}$ o custo para os demais itens da planilha foi $\mathrm{R} \$ 1.667,54$.

Em consideração ao repasse do SUS aos hospitais governamentais, verificamos que para cirurgia de revascularização do miocárdio (cirurgia de coronária com extra-corpórea), o repasse é de $R \$ 2.125,91$, excluindo UTI e material de perfusão que é pago à parte pelo SUS. O custo máximo repassado de material de perfusão é $\mathrm{R} \$ 1.575,48$ e o custo médio encontrado no nosso estudo foi de $R \$ 1.05 I, 24$, podendo-se observar que o repasse do SUS cobre os custos com perfusão. De $R \$ 2 .|25,9|$ que é o valor repassado pelo SUS para serviços hospitalares subtrai-se $\mathrm{R} \$ 1.667,54$, que foi o custo médio identificado dos demais materiais utilizados na cirurgia de revascularização do miocárdio, sobrando apenas $\mathrm{R} \$ 458,37$ para cobrir os demais serviços hospitalares referentes à internação, tais como: taxas, diárias, materiais e medicações para um período de permanência de sete dias, o que corres ponde a uma diária de $\mathrm{R} \$ 65,48$.

É importante lembrar que não foram considerados, para efeito do cálculo do custo da cirurgia, os custos indiretos e diretos com recursos humanos e não foram incluídos outros itens de materiais tais como: instrumentais cirúrgicos e roupas esterilizadas que dependeriam de cálculos de rateio de outras unidades da instituição.

É essencial destacar que a elaboração desta pesquisa permitiu um trabalho de conscientização da equipe cirúrgica, anterior à coleta de dados e no decorrer da pesquisa, possibilitando um envolvimento da equipe que passou a se preocupar mais com o controle de material.

As instituições de saúde não devem esquecer o importante papel de todos os seus colaboradores que compõem a organização, lembrando' que o sucesso no gerenciamento de custos depende da conscientização de todas as áreas envolvidas, do apoio da direção e mobilização de todos na busca de melhores indicadores de desempenho, reafirmando que o esforço do controle de custo é relevante à sobrevivência de hospitais que dependem da forma acentuada das (em geral) minguadas receitas geradas na prestação de serviços dos Institutos de saúde pública ou pelo SUS e, por isso, demonstra a importância das medidas de controle de material, que evitam desperdícios e fazem com que as instituições, que trabalham com esses repasses peloSUS, sobrevivam e possam apresentar uma posição salutar que permita seu equilíbrio e atualização tecnológica'.

\section{Conclusões}

I. O custo direto do material utilizado em cirurgia de revascularização do miocárdio apresentou uma média de $\mathrm{R} \$ 2.718,78$ ou US\$1329,35.

2. 0 custo direto do material utilizado em cirurgia de revascularização do miocárdio apresentou uma média, por número de pontes de safena, de:

- $\mathrm{R} \$ 2.207,7 \mathrm{I}$ ou US\$1079,45 para cirurgia de I ponte de safena;

- $\mathrm{R} \$ 2.554,6 \mathrm{I}$ ou US\$1249,07 para cirurgia de 2 pontes de safena;

- $\mathrm{R} \$ 2.768,94$ ou US\$1353,87 para cirurgia de 3 pontes de safena;

- $\mathrm{R} \$ 2.848,65$ ou US\$1392,84 para cirurgia de 4 pontes de safena;

- $R \$ 2.884,13$ ou US\$1410,19 para cirurgia de 5 pontes de safena.

3. Ficou demonstrado o aumento do custo da cirurgia, de acordo com o aumento do número de pontes de safena realizadas, variando em $R \$ 346,90$ a diferença entre as cirurgias de I a 2 pontes, $R \$ 2 \mid 4,33$ entre as cirurgias de 2 a 3 pontes, $R \$ 79,7$ I entre as cirurgias de 3 a 4 pontes e $R \$ 35,49$ entre as cirurgias de 4 a 5 pontes de safena.

4. Os materiais de perfusão ( $P$ ), fios cirúrgicos ( $\mathrm{FC}$ ) e de consumo (MC) apresentaram, respectivamente, os custos mais elevados.

5. Pode-se verificar que os custos médios dos itens perfusão $(\mathrm{P})$, fios cirúrgicos $(\mathrm{FC})$, gases $(G)$, equipamentos $(E Q)$ e medicações (M) se comportaram como custos variáveis, pois variam com $o$ aumento no número de pontes de safena realizadas.

6. Pode-se verificar que os custos médios dos itens material de consumo (MC), soros $(S)$, soluções $(\mathrm{SL})$ e derivados de sangue (DS) tiveram um comportamento assemelhado ao de custos fixos, pois suas variações não apresentam uma relação nítida com o aumento na variável número de pontes de safena realizadas.

\section{SUMMARY \\ Direct mean cost of the material USED IN CORONARY ARTERY BYPASS GRAFT SURGERY.}

PURPOSE. To verify the direct average cost of the material used in the Coronary Artery Bypass Graft (CABG) Surgery and to compare the average cost according to the number of bypasses perfomed (arterial and veins grafts).

Method. This is a descriptive study conducted in a Cardiac Surgery Center in São Paulo city. The sample includes 104 procedures for $C A B G$ surgery with extracorporeal circulation. The information was collected by a standard printed torn containing the material.

RESULTS. The analyses of the information showed that the direct mean cost of the material used in the surgery was $R \$ 2.718,78$.

Conclusions. A variation of material costs was observed proportional to the increase in number of bypasses performance, showing a statistical significant difference among surgerical interventions with I, 2 and 3 cal bypasses which was not observed in surgeries with 3, 4 and 5 bypases. The direct mean cost was: CABG with I bypass ( $R \$ 2.207,7 I), 2$ (R\$2.554,6I), 3 $(R \$ 2.768,94), 4(R \$ 2.848,65)$ and 5 $(R \$ 2.884,13)$. The items of the perfusion material $(R \$ 1.05 I, 24)$, surgical threads $(R \$ 829,98)$ and consumption material $(R \$ 442,40)$, represented those with a higher mean cost. [Rev Assoc Med Bras 2003; 49(3): 255-60]

KEY wORDS: Cardiac nursing. Cardiac surgery. Coronary artery bypass graft surgery. Costs. Medical economics. Health economics.

\section{ReFERÊNCIAS}

I. Beulke,R; Bertó,DJ. Gestão de custos e resultados na saúde. 2 ed. São Paulo, Saraiva, 2000.

2. Brunt,PD. Como reduzir custos: controlando gastos, eliminando desperdícios, trabaIhando com eficiência. Trad. de Sara Gedanke São Paulo, Nobel, 1992.

3. Couttolenc,Bf; Zucchi,P. Gestão de recursos financeiros. São Paulo, FSP/USP, I998. (Série Saúde \& Cidadania).

4. Silva,SH. da; Fernandes, Raq; Gonçalves, VLM. A administração de recursos materiais: importância do enfoque de custos e a responsabilidade dos profissionais de saúde. Rev. Bras. Enf., v.47, n.2, p.160-4, 1994 
5. Padilha,MICS. A qualidade da assistência de enfermagem e os custos hospitalares. Rev. Hosp. Adm. Saúde, v.14, n.3, p.128-33, 1990.

6. Ribeiro,FG. Analise dos custos direto no ato operatório de revascularização do miocárdio: insumos e categorias profissionais. São Paulo, 1998-11 Op. Dissertação (Mestrado)-Escola de Enfermagem, Universidade de São Paulo.

7. Beulke,R; Bertó,DJ. Gestão de custos e resultados na saúde: hospitais, clinicas, laboratórios e congêneres. São Paulo: Saraiva, 1997.

8. Neter,j; Wasserman, W; Kutner, M. H. Applied linear statistical models. 3ed. Homewood, IL, Irwin, 1990.
9. Soares,Jf; Siqueira, AL. Introdução à estatística médica. Belo Horizonte: Departamento de Estatística-UFMG, 1999.

Artigo recebido: $01 / 03 / 02$

Aceito para publicação: 01/04/03

\section{OPINIÃO DO LEITOR}

A nova Ramb abre espaço para o leitor na seção "Correspondências". Envie sua opinião ou sugestão sobre a revista ou artigos de seu interesse. Participe também enviando suas dúvidas ou sugestões sobre condutas práticas para a seção "À beira do leito". Sua colaboração é muito importante para aprimorarmos o nosso veículo de comunicação. Rua São Carlos do Pinhal, 324 Cep: 01333-903 - São Paulo - SP - E-mail:ramb@amb.org.br 